\title{
PROGRESS in Blood Pressure Control for the Prevention of Secondary Stroke
}

\author{
John Chalmers ${ }^{a}$ Stephen MacMahon ${ }^{b}$

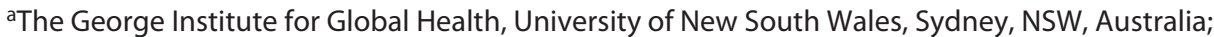 \\ bDepartment of Epidemiology and Biostatistics, Imperial College, London, UK
}

\section{Keywords}

Secondary stroke prevention · Blood pressure lowering ·

Perindopril and indapamide · Asian and Caucasian

participants

\section{Introduction}

The Perindopril Protection against Recurrent Stroke Study (PROGRESS) grew out of discussions at a World Health Organization/International Society of Hypertension (WHO-ISH) meeting on Hypertension and Cerebrovascular disease held in Nara, Japan, in May 1993 [1]. At the time, there was broad consensus that the benefits of blood pressure (BP) lowering were established for the primary prevention of stroke [2], but there was a clear need for further randomised trials to determine the role and possible benefits of BP lowering for the secondary prevention of stroke in patients who had survived a previous stroke as this remained an open question.

\section{Main Text}

During this initial meeting, there were numerous presentations on the epidemiology of stroke, and it became clear that both ischaemic and haemorrhagic stroke were much more frequent in the large populations of East Asia than in the Western populations in Europe and the USA, where the great majority of randomised trials of the risks and benefits of the long-term treatment of hypertension had been conducted. After the meeting, the 2 of us (Stephen MacMahon from the Clinical Trials Research Unit in Auckland and John Chalmers from the Flinders Medical Centre in Adelaide) agreed to work up a proposal and a protocol for a randomised control trial of BP lowering in patients with previous stroke. We also decided to include a significant proportion of patients from Asia so as to compare the outcomes in Eastern and Western populations, both from the point of view of establishing evidence in a broad range of patients and to determine the feasibility of conducting a trial in such geographically diverse regions. Accordingly, a draft protocol was drawn up, and a preliminary group of experienced clinical trialists from around the world was invited to meet and consider it at the 15th meeting of the ISH in Melbourne, Australia, in March 1994. karger@karger.com www.karger.com/ced
(C) 2021 S. Karger AG, Basel

Karger
Correspondence to:

John Chalmers, chalmers@ georgeinstitute.org.au 
At that meeting, the essential features of the protocol were confirmed, and it was agreed that the aim of the trial would be to provide reliable evidence about the balance of risks and benefits conferred by BP lowering achieved using an angiotensin-converting enzyme inhibitor-based regime, with or without an associated diuretic, in patients with cerebrovascular disease, with or without hypertension. The meeting agreed to invite professors Teruo Omae from Japan and Liu Lisheng from China to participate and join the Management Committee and to approach senior representatives of Servier International, who were attending the ISH meeting and ask them to consider supporting the trial. Thus, PROGRESS was born, supported by a substantial grant from Servier, with additional grants from the Health Research Council in New Zealand and the National Health and Medical Research Council in Australia [3]. The International Coordinating Centre was based in Auckland, with Regional Coordinating Centres in Beijing, Glasgow, Melbourne, Milan, Osaka, Paris, and Uppsala. Shortly after the launch of PROGRESS, clearer evidence was provided that BP lowering using the diuretic, indapamide, might reduce the risk of recurrent stroke by around 29\% in Chinese patients, but this was only a "preliminary report" [4] that was not confirmed until the publication of the definitive study in "Hypertension Research" in 2009 [5].

PROGRESS was a prospective, randomised, doubleblind, and placebo-controlled clinical trial conducted on subjects with previous stroke or transient ischaemic attack. After a 4-week active run-in phase, subjects with or without a history of hypertension received the ACE inhibitor perindopril $4 \mathrm{mg}$ daily and the diuretic indapamide $2.5 \mathrm{mg}$ daily or matching placebos, or alternatively perindopril alone versus matching placebo, at the discretion of the treating physician [6]. The primary outcome was total stroke, fatal or non-fatal, whether ischaemic or haemorrhagic, and secondary outcomes included fatal and disabling stroke, major vascular events (a composite of non-fatal stroke, non-fatal myocardial infarction, and cardiovascular death); cardiovascular death and all-cause death; dementia; and disability and dependency [6]. The required study sample size was estimated to be $6,000 \mathrm{pa}-$ tients, and the conduct of the study was overseen by an independent Management Committee that had no representatives of the study sponsors and 2 co-principal investigators, John Chalmers and Stephen MacMahon.

When the study started, Chalmers was in Adelaide as foundation professor and head of medicine at Flinders and MacMahon was in Auckland as director of the Clinical Trials Research Unit (CTRU). But during the life of the trial, Chalmers moved back to the University of Sydney as chairman of Research and Sub-Dean Research, based at the Royal North Shore Hospital in Sydney, and MacMahon and Robyn Norton, a well-established investigator in the field of injury prevention, decided to establish a new medical research institute, focusing on global health and non-communicable diseases, in a major university somewhere in the English-speaking world, and explored a variety of interested institutions. The strong collaboration between MacMahon and Chalmers, on the PROGRESS trial gave the University of Sydney a head start, and "The Institute for International Health" was born at that institution, with MacMahon and Norton as co-principal directors, on the first of January 1999, focussing initially on cardiovascular diseases and injury prevention. When the results of the PROGRESS trial were presented and published in 2001, the study gave the new institute an immediate and enviable reputation, and provided a very helpful means of advertising its arrival on the international stage. A few years later, the IIH became "the George Institute for Global Health" which it is today, now based at the University of New South Wales and has progressively broadened its range of interests.

The main results of PROGRESS were presented at the 2001 meeting of the European Society of Hypertension in Milan, with simultaneous publication in The Lancet [6]. A total of 7,121 potential participants were registered, of whom $14 \%$ were either found ineligible or withdrew from the 4-week active run-in on open-label perindopril $2 \mathrm{mg}$ daily followed by $4 \mathrm{mg}$ daily. Thus, 6,105 individuals entered the randomised, double-blind phase, with 3,051 and 3,054 on active treatment and placebo, respectively, including $58 \%$ on perindopril + indapamide and $42 \%$ on perindopril alone. There was good balance between the characteristics at baseline of participants on active treatment and those on placebo [7]. The overall mean BP was $147 / 86 \mathrm{~mm} \mathrm{Hg}$, and this was reduced by $9.0 / 4.0 \mathrm{~mm} \mathrm{Hg}$ in those assigned active treatment compared with those on placebo. The difference in BP reductions between active treatment and placebo groups was $12.3 / 5.0 \mathrm{~mm} \mathrm{Hg}$ among those on combination therapy but only $4.9 / 2.8$ $\mathrm{mm} \mathrm{Hg}$ among those on single drug therapy with perindopril alone [6].

There were 307 (10\%) participants in the active treatment group who had a stroke during follow-up, compared to $420(14 \%)$ in the placebo group, producing a relative risk reduction of $28 \%(p<0.0001$, shown in Fig. 1) [6]. There was no significant heterogeneity in the magnitude of the reduction in recurrent strokes defined by the nature of the qualifying event, or the geographic region 
Fig. 1. Major outcomes. Effects of study treatment on stroke subtypes, major vascular events, and deaths. Black squares = point estimates (with area proportional to number of events); horizontal lines $=95 \%$ CIs. Diamonds = point estimate and 95\% CI for overall effects. Vertical broken line $=$ point estimate for overall effect. $\mathrm{MI}=$ myocardial infarction. (Reprinted from PROGRESS Collaborative Group: Randomised trial of a perindopril-based BP-lowering regimen among 6,105 individuals with previous stroke or TIA. Lancet. 2001; 358(9287):1033-41. Fig. 4; Copyright 2001, with permission from Elsevier.) PROGRESS, Perindopril Protection against Recurrent Stroke Study; BP, blood pressure; TIA, transient ischaemic attack.

Fig. 2. Further analyses. Effects of study treatment on stroke and major vascular events in subgroups of patients. Hazard ratios (and 95\% CIs) for hypertensive and non-hypertensive subgroups standardised to study-wide proportions of patients for whom combination or single-drug therapy was planned. $p$ values for homogeneity (combination therapy vs. single-drug therapy) both $<0.001$. $p$ values for homogeneity (hypertensive vs. non-hypertensive) both $>0.6$. Conventions as in Fig. 1. (Reprinted from PROGRESS Collaborative Group: Randomised trial of a perindopril-based BP-lowering regimen among 6,105 individuals with previous stroke orTIA. Lancet. 2001;358(9287):1033-41. Fig. 5; Copyright 2001, with permission from Elsevier.) PROGRESS, Perindopril Protection against Recurrent Stroke Study; BP, blood pressure; TIA, transient ischaemic attack.

\begin{tabular}{|c|c|c|c|c|c|c|}
\hline & \multicolumn{3}{|c|}{ Number of events } & \multirow[b]{2}{*}{$\begin{array}{l}\text { Favours } \\
\text { active }\end{array}$} & \multirow[b]{2}{*}{$\begin{array}{l}\text { Favours } \\
\text { placebo }\end{array}$} & \multirow[b]{2}{*}{$\begin{array}{l}\text { Relative risk } \\
\text { reduction }(95 \% \mathrm{Cl})\end{array}$} \\
\hline & $\begin{array}{l}\text { Active } \\
(n=3,051)\end{array}$ & $\begin{array}{l}\text { Placeb } \\
(n=3,\end{array}$ & po & & & \\
\hline \multicolumn{7}{|l|}{ Stroke subtypes } \\
\hline Fatal or disabling & 123 & 181 & & & & $33 \%(15$ to 46$)$ \\
\hline Not fatal or disabling & 201 & 262 & & & & $24 \%$ (9 to 37 ) \\
\hline Ischaemic stroke & 246 & 319 & & & & $24 \%(10$ to 35$)$ \\
\hline Cerebral haemorrhage & 37 & 74 & $\leftrightarrow$ & & & $50 \%$ (26 to 67$)$ \\
\hline Stroke type unknown & 42 & 51 & & & - & $18 \%(-24$ to 45$)$ \\
\hline Total stroke & 307 & 420 & & & & $28 \%$ (17 to 38 ) \\
\hline \multicolumn{7}{|l|}{ Major vascular events } \\
\hline Vascular death & 181 & 198 & & & & $9 \%(-12$ to 25$)$ \\
\hline Non-fatal MI & 60 & 96 & & & & $38 \%(14$ to 15$)$ \\
\hline Non-fatal stroke & 275 & 380 & & & & $29 \%$ (17 to 39$)$ \\
\hline Total events & 458 & 604 & & & & $26 \%$ (16 to 34$)$ \\
\hline \multicolumn{7}{|l|}{ Mortality } \\
\hline Stroke & 42 & 50 & & & - & $16 \%(-27$ to 44$)$ \\
\hline Coronary & 58 & 62 & & & & $7 \%(-34$ to 39$)$ \\
\hline Other vascular & 81 & 86 & & & - & $6 \%(-28$ to 30$)$ \\
\hline Cancer & 64 & 65 & & & - & $2 \%(-39$ to 30$)$ \\
\hline Other non-vascular & 61 & 56 & & & $=$ & $9 \%(-57$ to 24$)$ \\
\hline \multirow[t]{3}{*}{ Total deaths } & 306 & 319 & & & $>$ & $4 \%(-12$ to 18$)$ \\
\hline & & & 0.5 & 1 & 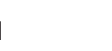 & 2 \\
\hline & \multicolumn{6}{|c|}{ Hazard ratio } \\
\hline
\end{tabular}

\begin{tabular}{|c|c|c|c|c|c|}
\hline & \multicolumn{2}{|c|}{ Events/patients } & \multirow{2}{*}{$\begin{array}{l}\text { Favours } \\
\text { active }\end{array}$} & \multirow{2}{*}{$\begin{array}{l}\text { Favours } \\
\text { placebo }\end{array}$} & \multirow{2}{*}{$\begin{array}{l}\text { Relative risk } \\
\text { reduction }(95 \% \mathrm{Cl})\end{array}$} \\
\hline & Active & Placebo & & & \\
\hline \multicolumn{6}{|l|}{ Stroke subtypes } \\
\hline Combination & $150 / 1,770$ & $255 / 1,774$ & & & $43 \%$ (30 to 54$)$ \\
\hline Single drug & $157 / 1,281$ & $165 / 1,280$ & & - & $5 \%(-19$ to 23$)$ \\
\hline Hypertensive & $163 / 1,464$ & $235 / 1,452$ & & & $32 \%(17$ to 44$)$ \\
\hline Non-hypertensive & $144 / 1,587$ & $185 / 1,602$ & & & $27 \%$ (8 to 42 ) \\
\hline Total stroke & $307 / 3,051$ & $420 / 3,054$ & & & $28 \%$ (17 to 38$)$ \\
\hline \multicolumn{6}{|c|}{ Major vascular events } \\
\hline Combination & $231 / 1,770$ & $367 / 1,774-$ & & & $40 \%$ (29 to 49$)$ \\
\hline Single drug & $227 / 1,281$ & $237 / 1,280$ & & & $4 \%(-15$ to 20$)$ \\
\hline Hypertensive & $240 / 1,464$ & $331 / 1,452$ & & & $29 \%$ (16 to 40$)$ \\
\hline Non-hypertensive & $218 / 1,587$ & $273 / 1,602$ & & & $24 \%$ (9 to 37$)$ \\
\hline \multirow[t]{3}{*}{ Total events } & $458 / 3,051$ & $604 / 3,054$ & & & $26 \%$ (16 to 34$)$ \\
\hline & & 0.5 & 1 & 1 & 2 \\
\hline & \multicolumn{5}{|c|}{ Hazard ratio } \\
\hline
\end{tabular}


of residence (Asia or elsewhere) [6, 8-10]. Fewer patients on active treatment had strokes that were fatal or disabling, and fewer patients on active treatment had either an ischaemic stroke (24\% reduction) or a haemorrhagic stroke, with a major $50 \%$ reduction compared to placebo in the latter [6]. Major vascular events were also reduced by $26 \%$, but mortality was not (shown in Fig. 1). There were similar reductions in both recurrent stroke and major vascular events in the hypertensive and non-hypertensive subgroups [6]. (shown in Fig. 2). While both these outcomes were significantly reduced in patients on combination therapy, among whom there was a particularly large $(12 / 5 \mathrm{~mm} \mathrm{Hg})$ reduction in $\mathrm{BP}$, they were not reduced in patients on single therapy, among whom there was a much smaller reduction $(5 / 3 \mathrm{~mm} \mathrm{Hg})$ in the active treatment group than placebo [6] (shown in Fig. 2).

Subsequent analyses published in the European Heart Journal demonstrated that active treatment with perindopril, plus or minus indapamide at the discretion of the treating physician, reduced the risk of both major coronary events and of congestive heart failure, each by $26 \%$ [11]. Another set of analyses examined the effects of active treatment with perindopril plus or minus indapamide at the discretion of the treatment physician, on the risks of dementia (defined using the DSM-IV criteria, as confirmed by a specialist, expert in the diagnosis of dementia) and cognitive decline (a decline of 3 or more points in the Mini-Mental State Examination [MMSE] score). These analyses confirmed reductions of $34 \%$ and $45 \%$, respectively, for dementia and cognitive decline associated with recurrent stroke, with no clear effect on either in the absence of recurrent stroke [12]. The PROGRESS trial also demonstrated that among individuals with cerebrovascular disease, the perindopril-based BP-lowering regimen not only reduced the risk of stroke and major vascular events but also substantially reduced the risk of the associated long-term disability, defined as a Barthel Index score of $<99 / 100$ and of dependency defined as a positive response to the question "In the last 2 weeks has the patient required regular help with every day activities"? [13].

Needless to say, we had some anxieties during the conduct of the trial. The first was that we had never worked with investigators from China or Japan and were a little anxious concerning the conduct of the study in those 2 study regions. But we need not have worried as our experience turned out to be extremely positive. Both were excellent in recruitment, with China under Liu Lisheng's leadership contributing 1,520 patients from 26 centres and Japan under Teruo Omae's leadership contributing
815 randomised patients from 33 centres. And both provided very high-quality data, with excellent performance in meeting all our quality benchmarks, such as the error rates on data entry, and in meeting the required timelines for a wide variety of study reports. We were also a little concerned before the start, lest CAT scans (which were requited for all randomised patients), might be more difficult to obtain in the Chinese centres. But as things turned out, these scans turned out to be much more freely available in China than in the UK, especially in the smaller or more peripheral centres. And of course, we were disappointed that the reduction in stroke in patients receiving perindopril alone was not significant, though the confidence limits were wide; and we were very pleased to see the very strong and significant reductions obtained for total stroke (43\%) and major vascular events (40\%) with the combination of perindopril and indapamide (shown in Fig. 2), which has become 1 of the favoured regimens for all patients with recurrent stroke and is increasingly recommended in all guidelines for hypertension now that most of these recommend starting treatment of hypertension with a combination of 2 first-line drugs.

Publication of the main results of PROGRESS 20 years ago, as well as the large volume of subsequent publications and reports, has exerted a major impact on the management of patients with stroke, reflecting the impressive reduction in both relative and absolute risks observed in patients on combination therapy with perindopril and indapamide. The results demonstrated that active treatment with this combination would result in avoiding 1 fatal or non-fatal vascular event (non-fatal stroke, nonfatal myocardial infarction, and vascular death), for every 11 patients assigned to this therapy. As a result, the major guidelines in Europe and the USA, and the guidelines of the WHO-ISH all recommended active treatment to lower BP in patients with previous stroke, a message that has been influential around the world [14-16] and particularly important for the prevention of ischaemic stroke, which was reduced by $24 \%$, allaying the fears of many neurologists and opinion leaders that reducing BP in such patients would exacerbate the ischaemic penumbra $[6$, 12]. Moreover, PROGRESS was the first major study to demonstrate reductions in haemorrhagic strokes, although the numbers were relatively small $[6,12]$, and confirmed the safety and benefits of BP lowering in patients with stroke whether hypertensive or not (shown in Fig. 2) [6] and in both Asian and Western populations [6, $9,10]$. Overall, PROGRESS established the benefits and safety of BP lowering for the secondary prevention of stroke and of other serious vascular events/diseases in pa- 
tients with previous stroke of various subtypes, among various ethnic groups, and across several major geographic regions. This treatment has now become an essential component in the fight to reduce the global burden of stroke and of cardiovascular disease across the world.

\section{Acknowledgements}

This article was published in celebration of the 30th Anniversary of the inception of Cerebrovascular Diseases 1991-2021.

\section{Conflict of Interest Statement}

Both authors report research grants from Servier for the PROGRESS and ADVANCE Trials and from the National Health and Medical Research Council for both studies.

\section{Funding Sources}

The authors did not receive any specific funding for the preparation of this manuscript.

\section{Author Contributions}

Both authors were originally involved in conceiving, planning, obtaining funding, and organising the original PROGRESS trial, and both contributed equally to the writing of this manuscript.

\section{Data Availability Statement}

This manuscript does not contain any original data but refers to the original publications of the PROGRESS Trial results in The Lancet [6] and a variety of other publications over many years. All reasonable requests for data sharing or collaboration will be considered.

\section{References}

1 Chalmers JP, Gyarfas I. In: Omea T, editor. Proceedings of WHO-ISH Meeting on Hypertension and Cerebrovascular Disease: Nara, Japan, 1993 May 25-27. Vol. 17: Hypertension Research. Japanese Society of Hypertension;1994. p. 1-119.

2 Collins R, Peto R, MacMahon S, Hebert P, Fiebach NH, Eberlein KA, et al. Blood pressure, stroke, and coronary heart disease. Part 2, short-term reductions in blood pressure: overview of randomised drug trials in their epidemiological context. Lancet. 1990;335: 827-38.

3 Neal B, MacMahon S. PROGRESS (perindopril protection against recurrent stroke study): rationale and design. PROGRESS management committee [corrected]. J Hypertens. 1995;13:1869-73. Erratum in: J Hypertens. 1996 Apr;14(4):535.

4 PATS Collaborative Group. Post-stroke antihypertensive treatment study. A preliminary report. Chin Med J. 1995;108(9):710-7.

5 Liu L, Wang Z, Gong L, Zhang Y, Thijs L, Staessen JA, et al. Blood pressure reduction for the secondary prevention of stroke: a Chinese trial and a systematic review of the literature. Hypertens Res. 2009;32:1032-40.

6 PROGRESS Collaborative Group. Randomised trial of a perindopril-based bloodpressure-lowering regimen among 6,105 individuals with previous stroke or transient ischaemic attack. Lancet. 2001;358:1033-41.
7 PROGRESS Management Committee (Chalmers J, Chairman). PROGRESS - perindopril protection against recurrent stroke study: characteristics of the study population at baseline. J Hypertens. 1999;17:1647-55.

8 Chapman N, Huxley R, Anderson C, Bousser MG, Chalmers J, Colman S, et al. Effects of perindopril-based blood pressure lowering regimen on the risk of recurrent stroke according to stroke subtype and medical history: the PROGRESS trial. Stroke. 2004;35:116-21.

9 Rodgers A, Chapman N, Woodward M, Liu LS, Colman S, Lee A, et al. Perindopril-based blood pressure lowering in individuals with cerebrovascular disease: consistency of benefits by age, sex and region. J Hypertens. 2004; 22:653-9.

10 Arima H, Anderson C, Omae T, Liu L, Tzourio $\mathrm{C}$, Woodward $\mathrm{M}$, et al. Effects of perindopril-based blood pressure lowering reduces major vascular events in Asian and Western subjects with cerebrovascular disease: the PROGRESS trial. J Hypertens. 2010;28:395400.

11 Chaturvedi V, Patel A, Chalmers J, Chapman N, Hansson L, MacMahon S, et al. Effects of a perindopril-based blood pressure lowering regimen on cardiac outcomes among patients with cerebrovascular disease. Eur Heart J. $2003 ; 24: 475-84$.
12 Tzourio C, Anderson C, Chapman N, Woodward M, Neal B, MacMahon S, et al. Effects of blood pressure lowering with perindopril and indapamide therapy on dementia and cognitive decline in patients with cerebrovascular disease. Arch Intern Med. 2003;163:1069-75.

13 Fransen M, Anderson C, Chalmers J, Chapman N, Davis S, MacMahon S, et al. Effects of a perindopril-based blood pressure-lowering regimen on disability and dependency in 6,105 patients with cerebrovascular disease. A randomised controlled trial. Stroke. 2003;34: 2333-8.

14 Kaplan N, Mendis S, Poulter N, Whitworth J. 2003 World Health Organization (WHO)/International Society of Hypertension (ISH) statement on management of hypertension. J Hypertens. 2003;21:1983-92.

15 Chobanian AV, Bakris GL, Black HR, Cushman WC, Green LA, Izzo JLJr, et al. Seventh report of the joint national committee on prevention, detection, evaluation, and treatment of high blood pressure. Hypertens. 2003;42: 1206-52.

16 European Society of Hypertension-European Society of Cardiology Guidelines Committee. 2003 European Society of Hypertension-European Society of Cardiology guidelines for the management of arterial hypertension. J Hypertens. 2003;21:1011-53. 